\title{
Gödel Is Right: There Is No Quantum Gravity
}

\author{
Ronald L. Huston \\ Department of Mechanical and Materials Engineering, University of Cincinnati, Cincinnati, USA \\ Email: ron.huston@uc.edu
}

How to cite this paper: Huston, R.L. (2018) Gödel Is Right: There Is No Quantum Gravity. Journal of Applied Mathematics and Physics, 6, 979-981. https://doi.org/10.4236/jamp.2018.65084

Received: April 18, 2018

Accepted: May 14, 2018

Published: May 17, 2018

Copyright $\odot 2018$ by author and Scientific Research Publishing Inc.

This work is licensed under the Creative Commons Attribution International License (CC BY 4.0).

http://creativecommons.org/licenses/by/4.0/

\begin{abstract}
Using two mathematical models, this paper points out fundamental differences between gravity and the other three fundamental forces (strong and weak nuclear forces and electromagnetic forces). These differences make untenable a reconciliation between gravity and the other forces. In conclusion, there is no quantum gravity and consequently, no gravitation-like particles.
\end{abstract}

\section{Keywords \\ Gravity, Quantum Gravity, Geometry}

\section{Introduction}

For many years, physicists, mathematicians, and others have attempted to construct a "theory of everything". From a physics perspective the focus is on reconciling gravity forces with the weak and strong nuclear forces and electromagnetic forces [1].

Some have argued that Gödel's theorem makes a theory of everything impossible to attain [2] [3]. The following paragraphs provide a supporting argument.

Many insightful and ingenious reconciliation analyses, models, and other formations have been prepared by physicists and mathematicians. Two of the most prominent models employ either string theory (with extra dimensions), or quantum-loop gravity.

Encountered difficulties appear to occur since gravity is space (or geometric) based while the other three are distinct particle based.

Interestingly the late Stephen Hawking originally believed that gravity could be reconciled with the other three fundamental forces, but he later abandoned that thought due to Gödel's theorem [4] [5]. 
Figure 1. Two lines: the proverbial "dotted line”, and a solid line.

\section{Analysis}

Space is a continuum but particles are discrete: Consider the set of all the positive numbers (the rational and the irrational). Let them be aligned in a row ascending according to their values. If we select any two numbers, arbitrarily close to each other, there will still be an infinite set of numbers between them. As long as the two numbers have distinct values, no matter how small their value difference, the numbers will still not contact each other. That is, the row is a discontinuous set.

Alternatively, consider the real line $R: R$ is a continuum. Selecting any two points, arbitrarily close to each other, there will still remain a segment of $R$ connecting the points. That is, $R$, is continuous.

Finally, as an illustration consider Figure 1 showing two representations of a line. The top line is composed of dots closely placed together. But since "dots" have zero dimension, there could be placed an infinite number of dots between any two adjacent dots. The denser the placement of the dots the more the dotted line will look like the solid line. But since the dotted line is always going to be discontinuous it will never become a solid line. That is, the dotted line and the solid line are irreconcilable.

\section{Discussion}

Attempting to reconcile gravity with the three particle forces is analogous to attempting to reconcile analog and digital representations of physical phenomenon. The conundrum is similar to an attempt to develop a catalog of all catalogs. The title "Catalog" is not in the listing and therefore the listing is incomplete. Alternatively, if "Catalog" is in the listing we do not have a name, or subject, of the envisioned construction. Similarly, a statement on a page saying: "This page is intentionally left blank" is a contradiction. In like manner, a "Theory of Everything" (ToE) would in itself be a contradiction.

\section{Conclusion}

There is no quantum gravity and therefore also no gravitons.

\section{Postword}

These remarks are not intended to disparage the extensive reconciliation work of others. Indeed, the results of those efforts have given us new insight into the nature of our universe and other fundamental understanding of forces and matter itself.

\section{References}

[1] Carlip, S. (2001) Quantum Gravity: A Progress Report. Reports on Progress in Physics, 64, 885-942. https://doi.org/10.1088/0034-4885/64/8/301 
[2] Robertson, D.S. (2000) Gödel's Theorem, the Theory of Everything, and the Future of Science and Mathematics. Complexity, 5, 22-27.

https://doi.org/10.1002/1099-0526(200005/06)5:5<22::AID-CPLX4>3.0.CO;2-0

[3] Feferman, S. (2006) The Nature and Significance of Gödel's Incompleteness Theorems. Institute for Advanced Study, Princeton, Gödel's Centenary Program, 1-16.

[4] Wikipedia (2018) Theory of Everything.

[5] Hawking, S. (2002) Gödel and the End of Physics. http://www.damtp.cam.ac.uk/strings02/dirac/hawking/ 